\title{
Antihypoxic oxygenation agents with respiratory hyperoxia to improve cancer immunotherapy
}

\author{
Stephen M. Hatfield and Michail V. Sitkovsky \\ New England Inflammation and Tissue Protection Institute, Department of Pharmaceutical Sciences and Biology, Northeastern University, Boston, Massachusetts, USA.
}

\begin{abstract}
Hypoxia/HIF-1 $\alpha$ - and extracellular adenosine/A2 adenosine receptor-mediated immunosuppression protects tissues from collateral damage by antipathogen immune cells. However, this mechanism also protects cancerous tissues by inhibiting antitumor immune cells in hypoxic and extracellular adenosine-rich tumors that are the most resistant to current therapies. Here, we explain a potentially novel, antiimmunosuppressive reasoning to justify strategies using respiratory hyperoxia and oxygenation agents in cancer treatment. Earlier attempts to use oxygenation of tumors as a monotherapy or to improve radiotherapy have failed because oxygenation protocols were not combined with immunotherapies of cancer. In contrast, the proposal for therapeutic use of antihypoxic oxygenation described here was motivated by the need to prevent the hypoxia/ HIF-1 $\alpha$-driven accumulation of extracellular adenosine to (a) unleash antitumor immune cells from inhibition by intracellular CAMP and (b) prevent immunosuppressive transcription of cAMP response element- and hypoxia response elementcontaining immunosuppressive gene products (e.g., TCF- $\beta$ ). Use of oxygenation agents together with inhibitors of the A2A adenosine receptor may be required to enable the most effective cancer immunotherapy. The emerging outcomes of clinical trials of cancer patients refractory to all other treatments provide support for the molecular and immunological mechanismbased approach to cancer immunotherapy described here.
\end{abstract}

\section{Introduction}

It has long been recognized that tumors are immunosuppressive, which explains the peaceful coexistence of tumors and tumorreactive immune cells in the same cancer patient (the Hellstrom paradox) and why durable responses are observed only in a minority of cancer immunotherapy patients $(1,2)$. Inspired by this paradox, our group's research has focused on solving this major problem, which led to uncovering a fundamental biochemical immunosuppressive mechanism that protects vital organs from collateral damage by antipathogen immune responses (3) and protects cancerous tissues from antitumor immune responses (4). In this Review, we summarize our studies of hypoxia/A2adenosinergic immunosuppression, which have been confirmed and extended by several other groups, leading to the current clinical trials of anti-hypoxia/A2-adenosinergic immunotherapies of cancer. These trials have shown promising outcomes by preventing inhibition of endogenously developed or immunotherapyenabled tumor-reactive immune cells $(5,6)$. In order to further improve cancer immunotherapy, we emphasize the advantages of the combination of oxygenation agents and respiratory hyperoxia

Conflict of interest: MVS is an inventor and author on a patent awarded in the United States, European Union, Australia, and Canada that belongs to the NIH and US Government (patent no. US8716301B2; "Methods for using extracellular adenosine inhibitors and adenosine receptor inhibitors to enhance immune response and inflammation") and claims the combination of anti-hypoxia/adenosinergic treatments with T cell-based immunotherapies of cancer. MVS received research funding and income in the form of salary from Juno Therapeutics. MVS has financial interest in the private companies Taurgen LLC and Oxymmune LLC.

Copyright: (5) 2020, American Society for Clinical Investigation.

Reference information: J Clin Invest. 2020;130(11):5629-5637.

https://doi.org/10.1172/JCl137554. as a treatment to reduce tumor hypoxia and weaken hypoxia/ HIF-1 $\alpha$ /A2-adenosinergic immunosuppression $(6,7)$.

Impaired oxygen delivery into poorly vascularized tumors and enhanced oxygen consumption by highly metabolic tumor cells account for the tumor hypoxia and stabilization of hypoxiainducible factor- $1 \alpha$ (HIF-1 $\alpha$ ) observed in solid tumors. Although HIF- $1 \alpha$ mediates transcriptional changes that allow tumor cells to adapt and thrive in a hypoxic tumor microenvironment (TME), it also promotes the upregulation of adenosine-generating enzymes (e.g., CD39/CD73) that ultimately strengthen suppression via the hypoxia/adenosinergic signaling axis (refs. 8-10 and Figure 1A). The connection between this immunosuppressive signaling pathway and tumor biology is also supported by demonstrations that targeting the A2B adenosine receptor (A2BR) can eradicate breast cancer stem cells (11). The promise of such adenosine-targeting drugs stems from the proof of principle provided in ongoing clinical trials of refractory cancer patients treated with A2A adenosine receptor (A2AR) antagonists to block the downstream stage of hypoxia/A2-adenosinergic immunosuppression $(5,6)$.

Until the discovery of immunological negative regulators (1214) and our discovery of hypoxia/A2-adenosinergic immunosuppression $(3,4,10)$, it was unclear what in the TME prevented hundreds of millions of adoptively transferred $\mathrm{T}$ cells from destroying tumors in vivo $(7,15,16)$.

Our hope was that defining the biochemical inhibitory mechanism would enable identification of druggable molecular targets. Targeting these biochemical inhibitory mechanisms would allow the elimination of immunosuppression and tumor rejection by tumor-reactive $\mathrm{T}$ cells, especially if combined with blockade of immunological negative regulators (e.g., CTLA-4/PD-1) to prevent $\mathrm{T}$ cell inhibition and exhaustion. Therapeutic antibodies 

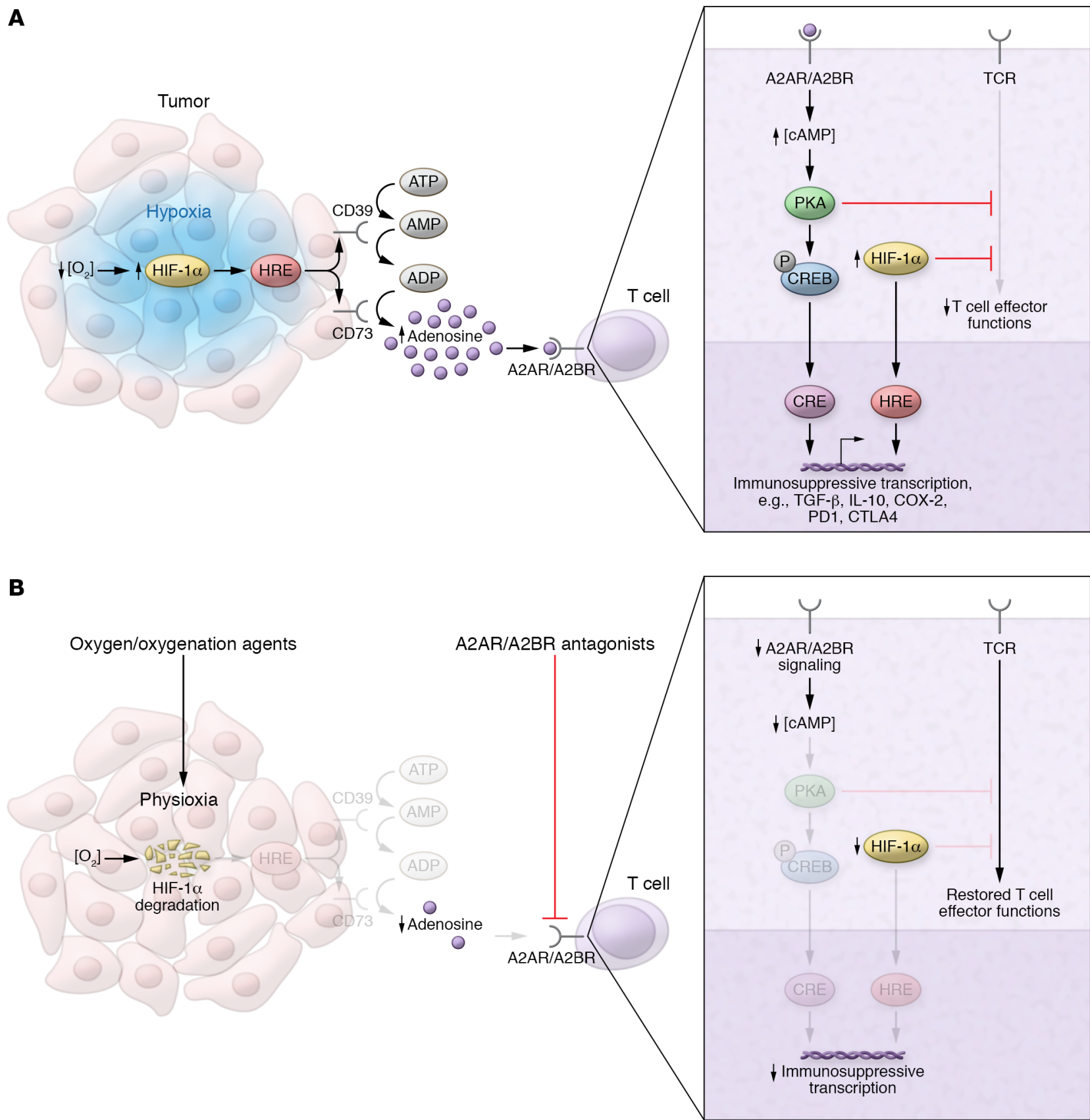

Figure 1. Immunosuppressive hypoxia/adenosinergic pathway in tumors and strategies for therapeutic targeting. (A) Tumor hypoxia-driven accumulation of extracellular adenosine inhibits tumor-reactive T cells via A2A/A2B adenosine receptors. Tumor hypoxia (blue) stabilizes HIF-1 $\alpha$, increasing transcription of adenosine-generating ectoenzymes (e.g., CD39 and CD73), whose gene promoters contain hypoxia response elements (HREs). Adenosine binds to cAMP-elevating A2AR/A2BR on the surface of T cells, initiating protein kinase A-mediated (PKA-mediated) signaling cascades and resulting in the inhibition of T cell effector functions and immunosuppressive transcription via cAMP response elements (CREs). HIF-1 $\alpha$ may synergize with cAMP signaling by directly inhibiting T cell effector functions and promoting immunosuppressive transcription via HREs. (B) Strategies targeting upstream and downstream stages of hypoxia/A2-adenosinergic immunosuppression in the TME. Oxygen/oxygenation agents restore physiological oxygen levels (physioxia) within the tumor, leading to oxygen-dependent degradation of HIF-1 $\alpha$. At the level of T cells, oxygenation therapy-induced reductions in HIF- $1 \alpha$ also promote restoration of effector functions. A2AR/A2BR antagonists block the adenosinergic signaling that triggers CRE-mediated immunosuppressive transcription.

blocking immune checkpoints have dramatically improved the treatment of previously refractory cancer patients with diverse malignancies (16); however, clinical outcomes of immune checkpoint inhibition in patients with solid tumors other than melanoma have been disappointing, for at least two major reasons. First, some cancer patients do not possess sufficient numbers of anti- tumor immune cells, which may result in immunologically "cold" tumors. This may be explained by our observation that antitumor T cells avoid hypoxic TMEs $(8,17)$.

Second, even if cancer patients do possess a sufficient number of intratumoral tumor-reactive effector T and NK cells (i.e., immunologically "hot" tumors), these cells may still be inhibited 
by biochemical negative regulators of the immune response that operate in the most treatment-resistant and hostile TMEs, which are hypoxic and extracellular adenosine-rich (7). Therefore, tumor-reactive $\mathrm{T}$ or NK cells will still be inhibited by hypoxia and adenosine even after anti-PD-1/anti-CTLA-4 therapy (7). Indeed, tumor hypoxia-driven accumulation of immunosuppressive adenosine may contribute to $\mathrm{T}$ cell exhaustion and impaired cytotoxicity, cytokine secretion, and tumor infiltration (refs. 4, 8-10, 18-21, and Figure 1A).

Virtually all known T cell- and NK cell-based cancer immunotherapies are susceptible to hypoxia/adenosinergic immunosuppression, including adoptive cell therapy (ACT), cancer vaccines, immunostimulating adjuvants, chemoimmunotherapy, and radioimmunotherapy as well as therapeutic targeting of other immune checkpoints $(16,22)$. Among these immunotherapies, FDAapproved blockade of CTLA-4 and of PD- 1 has been the most promising. Chimeric antigen receptor T cell (CART) therapy has also received regulatory approval for the targeting of CD19 for B cell lymphoma. ACT of CD19-targeting CARTs engineered from cancer patients' own polyclonal T cells against hematological cancers has demonstrated impressive clinical outcomes by recognizing and eliminating tumor targets. Nevertheless, CART therapy's success has not been extended from hematological malignancies to solid tumors (23), where the hypoxic TME provides tumor protection against CARTs.

Thus, we have promoted a therapeutic strategy that may improve efficacy of any of these cancer immunotherapies by eliminating this powerful biochemical hypoxia/HIF-1 $\alpha$ /adenosine/ A2AR immunosuppressive barrier in the TME (Figure 1, A and B). We suggest that to maximize efficacy, combination immunotherapy must (a) target immunological negative regulators, (b) target biochemical negative regulators, and (c) ensure that patients have sufficient numbers of nonexhausted tumor-reactive T cells or NK cells. For example, this could be achieved by treating patients with anti-PD-1/anti-CTLA-4 antibody, anti-hypoxia/adenosinergic drugs, and ACT using T cells, NK cells, or CARTs. The success of this strategy is dependent on a more complete understanding of the mechanisms of $\mathrm{T}$ cell function - and dysfunction - in the hypoxic and adenosine-rich TME. Of crucial importance will be insights from single-cell analysis of the state of $\mathrm{CD}^{+} \mathrm{T}$ cells in human tumors (24).

We postulate that the most effective approach to target biochemical negative regulators would be to simultaneously treat patients with (a) the combination of oxygenation agents and respiratory hyperoxia to block hypoxia/HIF-1 $\alpha$-mediated immunosuppressive transcription, and (b) A2AR antagonists to block A2AR/ cAMP/protein kinase A-mediated immunosuppression. Translational studies of anti-hypoxia/A2-adenosinergic drugs for cancer immunotherapy have been initiated as a result of the recent understanding that the hypoxia/adenosine axis inhibits antitumor immune cells $(4,8)$, and the preexisting approach of targeting hypoxia-inducible factors (HIFs) in tumors (25). In this context, it would be appealing to repurpose HIF inhibitors - a fascinating field established by landmark studies from Semenza's group identifying HIFs and the importance of their therapeutic targeting in tumors (25). These studies were accompanied by complementary breakthroughs in understanding by Kaelin's and Ratcliffe's groups of the hypoxia- and oxygen-sensing mechanisms that control the stability and degradation of HIFs (26).

\section{Hypoxia/HIF-1 $\alpha$-mediated and A2-adenosinergic immunosuppression by delayed negative feedback}

We focus here on biochemical negative regulators since, as laws of physics govern chemical processes, so too might biochemical processes govern the recruitment of immunological negative regulators - especially regarding such fundamental molecules of life as oxygen and adenosine. Indeed, the hypoxia/HIF-1 $\alpha$ axis may be at the top of the pyramid of immunosuppressive mechanisms, since elimination by oxygenation weakens all other tested biochemical and immunological negative regulators $(8,9)$.

It is now established that hypoxia/HIF-1 $\alpha$ contributes to the accumulation of extracellular adenosine, which signals through cAMP-elevating A2AR/A2BR to suppress immune cell effector functions (Figure $1 \mathrm{~A}$ and reviewed in ref. 7). In addition to the direct inhibition of $\mathrm{T}$ cell receptor (TCR) signaling, HIF- $1 \alpha$ and cAMP create an immunosuppressive environment by activating immunosuppressive transcription via the hypoxia response element (HRE) and cAMP response element (CRE) (7-10). This cAMP/CREand HIF- $1 \alpha / \mathrm{HRE}$-driven transcription also ensures the recruitment of other negative regulators to maximize immunosuppression in inflamed and cancerous tissue microenvironments (Figure 1, A and B). Additionally, cAMP-dependent protein kinase A (PKA) can recruit the transcriptional activity of $\mathrm{HIF}-1 \alpha$, reflecting the existence of crosstalk between adenosine/A2AR/cAMP-mediated and HIF$1 \alpha$-mediated transcription (27). This concept of hypoxia/A2-adenosinergic immunosuppressive transcription is strongly supported by studies by Powell's and Haskó's groups, who were first to demonstrate the ability of A2AR-mediated signaling to increase immunological negative regulators, such as LAG3, IL-10, and Tregs $(18,28)$.

Figure $1 \mathrm{~B}$ identifies several druggable molecular targets in the hypoxia/A2-adenosinergic pathway. Many existing drugs are now being repurposed in order to inhibit the upstream, intermediate, and downstream stages and thereby weaken immunosuppression in tumors. In our original studies, in addition to A2AR and A2BR inhibitors, we also considered inhibitors of adenosine-generating enzymes and adenosine-degrading drugs (7). However, we decided to focus on A2AR inhibitors and oxygenation agents as the most feasible and mechanistically justified approach $(4,8,9,29)$. Indeed, synthetic oxygenation agents and blood substitutes have emerged as powerful tools to induce maximal tumor-rejecting capacity of cancer immunotherapies $(8,9)$.

Studies using $A 2 A R$ gene deletion in combination with systemic oxygenation in vivo $(8,9)$ established that the upstream hypoxia/HIF-1 $\alpha$ signaling is connected with the downstream A2AR/cAMP signaling via extracellular adenosine-generating enzymes. This antiinflammatory mechanism normally functions to protect hypoxic and adenosine-rich inflamed tissues of vital organs from excessive collateral damage during antipathogen immune responses. However, when the same pathway protects hypoxic and adenosine-rich cancerous tissues from attack by cytotoxic $\mathrm{T}$ lymphocytes, it is critical to weaken this response using drugs that (a) eliminate upstream hypoxia/HIF-1 $\alpha$ signaling or (b) block downstream adenosine/A2AR-mediated immunosuppression - both examples of therapeutic engineering of inflammation. 


\section{Pathophysiologically immunosuppressive extracellular adenosine/A2AR/cAMP axis}

Our search to identify physiological mechanisms that terminate inflammation began with the hypothesis that intracellular cAMP in $\mathrm{T}$ cells plays a key immunosuppressive role in vivo. This was supported by in vitro data showing the inhibition of many effector functions of T cells by cAMP-dependent PKA (30-34). The next step was to identify which among the many cAMP-elevating $G_{s}$ protein-coupled receptors (G PCRs) was involved in the inhibition of immune cells in vivo. We focused on extracellular adenosine because of its uniquely ubiquitous nature in cells and tissues among all other $\mathrm{G}_{\mathrm{s}}$ PCR ligands, and because it was assumed that adenosine can be formed rapidly by the enzymatic hydrolysis of ATP to adenosine (35-41).

Genetic and pharmacological evidence that A2ARs on immune cells have a critical role in attenuating inflammation was first reported in models of autoimmune and viral hepatitis and acute lung inflammation $(3,42)$. We demonstrated that inflammatory stimuli inducing only minimal inflammation in wild-type (WT) mice triggered much more severe inflammatory tissue damage and led to deaths of A2AR-deficient mice $(3,42)$. These studies firmly implicated A2AR as a critical negative feedback mechanism regulating inflammatory responses. A2AR was also demonstrated to be nonredundant, as control assays indicated that none of the other functional cAMP-elevating receptors were able to compensate for the loss of A2AR (3).

These data prompted important studies of extracellular adenosine-generating enzymes. The most influential were studies of CD39, pioneered by Robson's team $(43,44)$, and studies of CD73, pioneered by teams led by Smyth, and Zhang and Thompson (45, 46). CD39 and CD73 act in tandem upstream of adenosine receptors by generating extracellular adenosine. This important process providing protection from excessive inflammatory damage was shown to be mediated by downstream signaling via A2AR (3, 47-50). These considerations pointed to the need to identify and target the upstream stages of the hypoxia/adenosinergic pathway.

\section{Pathophysiologically immunosuppressive hypoxia/HIF-1 $\alpha$ axis}

We postulated that hypoxia/HIF-1 $\alpha$ signaling in inflamed or cancerous tissues would suppress immune responses as a result of (a) immunosuppressive transcription mediated by HIF- $1 \alpha$ signaling (10) independently of adenosine/cAMP and (b) the inhibition of TCR-triggered transmembrane signaling in T cells by intracellular cAMP elevated by extracellular adenosine-activated A2AR (Figure 1A). It is important to note that cAMP-mediated immunosuppression is driven by HIF- $1 \alpha$-mediated accumulation of extracellular adenosine. The connection between hypoxia and extracellular adenosine was first demonstrated in studies of the role of HIF- $1 \alpha$ in permeability changes in intestinal epithelia (51). These findings benefitted immunological research leading to the current views of the role of tumor hypoxia/HIF-1 $\alpha$ in the enhancement of CD39and CD73-dependent generation of extracellular adenosine.

Providing genetic evidence for such a role of HIF-1 $\alpha$ in immune cells was complicated owing to embryonic lethality of mice with total deletion of the HIF- $1 \alpha$ gene. To overcome this, we developed RAG-2-deficient blastocyst complementation
$\mathrm{Hifla}^{-/-} \rightarrow \mathrm{Rag2}^{-/-}$chimeric mice with T cell- and B cell-targeted deletion of HIF-1 $\alpha$ (52). The antiinflammatory functions of HIF$1 \alpha$ in vivo were reflected in observations of loss of control of inflammation and in dramatic autoimmunity in these chimeric mice (52). HIF-1 $\alpha$ deficiency in $\mathrm{T}$ and $\mathrm{B}$ cells also resulted in the appearance of abnormal peritoneal B-1-like lymphocytes with high expression of CD45, as well as abnormal maturation of B-2 lymphocytes in bone marrow (52). These studies demonstrated that when HIF- $1 \alpha$ is absent, there is severely impaired control of inflammation and autoimmunity.

In our continued studies of HIF-1 $\alpha$-mediated immunosuppression, we tested different, but complementary, genetically engineered mice with (a) deletion of activation-inducible I.1 isoform of HIF-1 $\alpha$, (b) T cell-targeted HIF-1 $\alpha$ deletion, or (c) classical HIF- $1 \alpha$ complete gene deletion in $\mathrm{T}$ and $\mathrm{B}$ lymphocytes using the RAG-2 blastocyst complementation system. In all three types of genetically altered mice, the deficiency of classical HIF- $1 \alpha$ or the I.1 isoform resulted in higher production of proinflammatory cytokines by TCR-activated HIF- $1 \alpha$-deficient T lymphocytes compared with HIF-1 $\alpha$-expressing WT T cells (53-58).

Consistent with HIF-1 $\alpha$ acting as a negative regulator of immune cells, the antibacterial activity of T cells was enhanced due to HIF-1 $\alpha$ knockdown. To avoid artifacts from artificial skewing of TCR repertoire in Hifla-deficient mice due to the likely involvement of HIF-1 $\alpha$ in negative and positive selection in the thymus, we evaluated the bacterial superantigen-mediated activation of polyclonal T cells, which is not dependent on the recognition of antigen by $\alpha \beta$ TCRs as in other models. These studies were performed in mice with deletion of Hifla in models of bacterial sepsis (58).

It is also possible to improve the antibacterial activity of $\mathrm{T}$ cells by targeting the HIF-1 $\alpha$ isoform I.1 in T cells (56). This targeting was accompanied by reduced bacterial load, increased M1 macrophage polarization, elevated proinflammatory cytokines, reduced levels of IL-10, and improved survival in a murine peritonitis model (57). We discovered this mRNA isoform of human HIF- $1 \alpha$ in $\mathrm{T}$ cells that is expressed using the alternative first exon I. 3 and is increased after TCR stimulation. Interestingly, the I.3 isoform of HIF- $1 \alpha$ is expressed in humans in a tissue-specific manner, with the highest expression found in peripheral blood leukocytes and thymus (56).

Taken together, these in vivo studies genetically targeting HIF- $1 \alpha$ in $\mathrm{T}$ cells provide strong molecular justification for the pharmacological inactivation of hypoxia/HIF-1 $\alpha$ as a therapeutic strategy - especially when the goal is better recruitment and stronger antipathogen/antitumor effector functions of $\mathrm{T}$ cells by prevention of inhibition in hypoxic tissues.

\section{Anti-A2A-adenosinergic immunotherapies of cancer using A2AR inhibitors}

Studies of the immunosuppressive role of hypoxia/HIF- $1 \alpha$ were performed in parallel with research on the downstream adenosine/ A2AR-mediated signaling. It was established in preclinical testing that the tissue-protecting adenosine/A2AR axis of immunosuppression also protects cancerous tissues by inhibiting antitumor $\mathrm{T}$ cells (4). Increased tumor rejection and survival were detected in A2AR-deficient mice as compared with WT mice. Tumor rejection was also observed in WT mice treated with synthetic or natu- 
ral A2AR antagonists (4). The tumor-protecting role of adenosine/ A2AR was further evidenced in the observations of enhanced tumor rejection by ACT of antitumor T cells deficient in A2AR, due to either $A 2 A R$ gene deletion or knockdown with siRNA (4, $59)$. These studies also provided an approach for manufacturing T cells for ACT: Better tumor rejection was observed in mice if tumor-reactive $\mathrm{T}$ cells deficient in or depleted of A2AR were selected $(59,60)$.

The assays described above were the first to demonstrate that the effects of A2AR were T cell-dependent and that targeting the hypoxia/A2A-adenosinergic pathway is an effective immunotherapeutic strategy to prevent inhibition of antitumor $\mathrm{T}$ cells in the TME. Importantly, effects of A2AR blockade were confirmed by teams led by Powell $(18,61)$, Smyth and Zhang, and others who have joined our appeals to use A2AR inhibitors in combination with cancer immunotherapies $(7,21,45,62,63)$. Interestingly, it was also shown that A2AR blockade enhances the antitumor effector functions of CARTs (64).

Convincing preclinical data (4) coupled with the lack of therapeutic options to treat cancer patients refractory to all other treatments stimulated the efforts of pharmaceutical companies and triggered the formation of clinical-stage start-ups to test synthetic inhibitors of A2AR and CD39/CD73 in the clinic (65). Companies including AstraZeneca and Corvus have further confirmed the effects of A2AR inhibitors in promoting tumor rejection in preclinical studies (66). Clinical trials are now reporting promising responses and tumor regression in patients with cancers that are otherwise refractory to all other treatments (e.g., renal cell cancer and metastatic castration-resistant prostate cancer).

A2AR inhibitors were shown to safely block adenosine signaling in patients, with clinical responses observed when the drug was used alone or in combination with anti-PD-L1. This study included subjects who were previously nonresponsive to PD-1/ PD-L1 inhibitors. Importantly, treatment with the A2AR inhibitor increased intratumoral recruitment of $\mathrm{CD}^{+} \mathrm{T}$ cells, which was associated with durable clinical responses. Of potential benefit to cancer patients was the identification of an adenosine-regulated gene expression signature in pretreatment tumor biopsies. This signature could serve as an inclusion criteria, or conversely as an exclusion criteria if the signature is not expressed in patients (6). Thus, the use of synthetic A2AR inhibitors in preclinical and clinical studies has provided proof of principle for the therapeutic use of these classes of drugs as potentially novel immunological coadjuvants $(3,4)$.

The evidence for therapeutic benefits of blocking the downstream end stage of the adenosine/A2AR axis is also predictive of the clinical efficacy of oxygenation agents that act upstream of A2AR in this pathway to reverse immunosuppression in tumors. In a recent review of the clinical trials using A2AR inhibitors, we proposed that even in the best-case scenario (i.e., adenosine-rich tumors with sufficient numbers of antitumor $\mathrm{T}$ and NK cells), there will still be tumor protection governed by HIF- $1 \alpha-$ and HREmediated immunosuppressive transcription (5). These insights have provided the rationale for combining A2AR inhibitors with tumor oxygenation to target the upstream hypoxia/HIF-1 $\alpha$ signaling in hypoxic and adenosine-rich tumors. The feasible and justifiable combination of these treatments is expected to completely eliminate the upstream and downstream stages of immunosuppression by unleashing the strongest possible antitumor response.

\section{Immunosuppressive versus T cell-metabolic effects of hypoxia/HIF-1 $\alpha$ axis}

Studies by our team uncovered the powerful immunosuppression by the HIF-1 $\alpha$-governed pathway (52). This served as the motivation to focus on studies of the elimination of hypoxia/HIF-1 $\alpha-$ mediated immunosuppression in tumors to enable immunotherapies of cancer and infectious diseases. It was instructive that insights about HIF- $1 \alpha$ were complementary to those in our studies of A2-adenosinergic immunosuppressive signaling $(3,4)$. This research is currently being translated into the clinic with the goal of preventing HIF- $1 \alpha$ from protecting hypoxic tumors from antitumor T and NK cells $(8,9)$.

It was recognized early in in vitro studies that hypoxia can have both inhibitory and enhancing effects on different functions of cytotoxic T lymphocytes (CTLs), and that the effects of hypoxia are time-dependent (65). Hypoxia, and by extension the hypoxia/ HIF-1 $\alpha$ axis, exerted immunosuppressive effects on T cells by inhibiting the accumulation of IL-2 and IFN- $\gamma$ and delaying the development of CTLs (67). This pointed toward the immunosuppressive capacities of hypoxia. However, the delayed development of CTLs by hypoxia ultimately resulted in more lytic cells (67), suggesting that hypoxia/HIF-1 $\alpha$ may not only inhibit some TCR-driven responses of $\mathrm{T}$ cells, but also may trigger transcription of some important CTL differentiation factors. This view is supported by the observation that hypoxic incubation and TCR-mediated activation of $\mathrm{T}$ cells were additive in enhancing levels of HREcontaining gene products like VEGF (67). Since the VEGF promoter also contains a CRE, hypoxia-generated VEGF may represent a feedback mechanism by which both hypoxia and cAMP mitigate hypoxia by increasing blood supply (10). In subsequent investigations we found that the immunosuppressive cytokine TGF- $\beta$, another CRE- and HRE-containing gene product, is strongly decreased after elimination of hypoxia by supplemental oxygenation (8). We concluded from these studies that cAMP/PKA/CRE and hypoxia/HIF-1/HRE signaling may create cold tumors that are reprogrammed toward immunosuppression by promoting the synthesis of many different immunosuppressive molecules (refs. 8, 10; Figure 1, A and B).

The differential effects of hypoxia may reflect the differentiation-stage-specific need for HIF- $1 \alpha$ in both T and B cells, though so far this has been established only for B cells using rigorous genetic controls (68). Comparison of WT and HIF- $1 \alpha$-deficient B220 ${ }^{+}$ bone marrow cells showed that HIF- $1 \alpha$ plays an important role in $B$ cell development and differentiation, and that glucose dependency differs at different B cell developmental stages (68).

The role of hypoxia in B cell function was further investigated in studies by Abbott et al. that were guided by the assumption that highly proliferating B cells outpace the development of the vascular bed, resulting in germinal center (GC) hypoxia (69). B cells may create a hypoxic microenvironment for metabolic reprogramming, suggesting that physiological hypoxia plays a critical role in GC B cell differentiation (69). Parallel studies by Boothby's group demonstrated that GC light zones are hypoxic and that low oxygen alters B cell physiology and function (70). More recently, using 
targeted elimination of HIF- $1 \alpha$ or both HIF- $1 \alpha$ and HIF- $2 \alpha$ in CD $4^{+}$ $\mathrm{T}$ cells, they showed the importance of hypoxia/HIFs in $\mathrm{T}$ cell help with antibody responses and antibody class switching (71). It would be interesting to reconcile some of the differences between these groups' results in studies of the effects of hypoxia/HIFs on B cells versus T cell help during time course analysis of GC and B cell development in genetically altered mice.

Important in vivo evidence that hypoxia is immunosuppressive was provided by demonstrations that $\mathrm{T}$ cells exposed to hypoxic environments in vivo are less activated (72). This was done by parallel analysis of $\mathrm{T}$ cells in the same mice exposed to higher (21\% oxygen) or lower (8\%) oxygen levels. Subsequent analysis revealed that the degree of $\mathrm{T}$ cell activation in vivo was significantly stronger in more oxygenated $\mathrm{T}$ cells. Taken together, these observations suggest that the extent of $\mathrm{T}$ cell activation in vivo is localization-dependent and is decreased in environments with low oxygen tension (72).

The antiinflammatory effects of hypoxia were also observed in studies of T cell-mediated hepatitis, demonstrating hypoxia functions upstream and A2AR functions downstream within the same immunosuppressive and liver tissue-protecting pathway (73). Similarly, the hypoxia/adenosine/A2BR pathway was shown to play a critical role in the prevention of liver ischemia/reperfusion injury (IRI) in vivo. Hypoxic activation of this pathway warranted the use of selective A2BR agonists or even intermittent hypoxia to protect from liver IRI (74). Finally, the negative regulation of T cell activation by HIF- $1 \alpha$ was also confirmed in studies of CD $4^{+} \mathrm{T}$ cells in models of diabetes (75).

Interestingly, it was observed that hypoxia-induced immunosuppression of T cells can also be A2AR-independent. However, this type of inhibition of $\mathrm{T}$ cells was shown to be short-lived and quickly reversible. T cells rapidly recovered the ability to produce IFN- $\gamma$ upon restimulation. These studies suggest that $\mathrm{T}$ cell inhibition by hypoxia is likely mediated by several distinct mechanisms (76).

\section{Hypoxia/HIF-1 $\alpha$ regulates T cell responses by altering metabolism}

In important parallel investigations using different approaches, Randall Johnson's team also investigated the role of HIF-1 $\alpha$ in T cells. In contrast to our focus on immunosuppression, Johnson and colleagues examined how to take advantage of important cell physiological functions of HIF- $1 \alpha$ in order to improve the antitumor qualities of T cells. Palazon et al. demonstrated that HIF- $1 \alpha$ is an essential regulator of $\mathrm{T}$ cell effector responses in the TME including differentiation, migration, and expression of costimulatory receptors (77). In accordance with our previous observations that more lytic CTLs developed in hypoxia (67), Johnson's group found that hypoxia increased the production of the cytolytic molecule granzyme B (77). Also in agreement with our hypothesis of HIF-1 $\alpha / \mathrm{HRE}-$ and cAMP/CRE-mediated immunosuppressive transcription $(7,10)$, Johnson's team found that hypoxia increased expression of the negative immunological regulators PD-1, TIM3, and LAG3 in a HIF- $1 \alpha$ - but not HIF- $2 \alpha$-dependent manner (77). The use of sophisticated genetic tools enabled this team to spotlight not just HIF- $1 \alpha$ alone, but specifically the HIF-1 $\alpha$ /VEGF-A pathway in T cells (77). Interestingly, in our earlier study (67), we also noticed the connection between hypoxia and VEGF and $\mathrm{T}$ cells, and that secretion of IFN- $\gamma$ was inhibited while secretion of
VEGF was promoted by hypoxia. It would be of important scientific merit to evaluate the similarities and reconcile the differences in the observations of the diverse roles of HIF- $1 \alpha$ in T cells obtained in different genetic models of HIF- $1 \alpha$ deletion by our two teams and following studies.

\section{Targeting immunosuppressive hypoxia/HIF-1 $\alpha$ axis}

In contrast to our basic reasoning, a major focus of cancer therapies has been on inhibiting HIF-1 $\alpha$ in tumor cells in an effort to promote tumor cell death (78). HIF-1 $\alpha$ is a well-appreciated target for cancer therapies, and drugs that inhibit hypoxia/HIF-1 $\alpha$ signaling are in high demand due to the previous understanding of the protumor effects of hypoxia $(78,79)$. Inhibitors of HIF- $1 \alpha$, such as digoxin and acriflavine, were shown to decrease lung metastasis in an orthotopic breast cancer model (78). However, until now, efforts to develop HIF-1 $\alpha$-targeting small molecules have been largely unsuccessful. Yet the hypoxia/HIF-1 $\alpha$ axis represents a feasible drug target for available and safe medical treatments such as supplemental oxygen and oxygenation agents $(8-10,80)$.

Here, we promote a new therapeutic paradigm in targeting HIF-1 $\alpha$ in cancer by suggesting that maximal tumor rejection following inhibition of hypoxia/HIF-1 $\alpha$ will occur only in combination with immunotherapies. While the tumor-protecting role of HIF- $1 \alpha$ is well established $(81,82)$, the more recent understanding that HIF-1 $\alpha$ is critical in orchestrating immunosuppression in the TME has led to the potentially novel motivation to inhibit HIF-1 $\alpha$ during the immunotherapy of cancer $(5,7-9,80)$. Accordingly, HIF- $1 \alpha$ inhibitors may reprogram an immunosuppressive TME into an immunopermissive TME, leaving the tumor immunologically defenseless. However, in the absence of tumor-reactive $\mathrm{T}$ cells, even an immunologically defenseless tumor will not disappear. Thus, inhibitors of HIF-1 $\alpha$ should be combined with immunotherapies that provide or enhance $\mathrm{T}$ and NK cell responses to ensure maximal tumor rejection.

\section{Anti-hypoxia/A2-adenosinergic motivation to use systemic oxygenation}

Based on our studies, we have long advocated for the therapeutic mitigation of hypoxia/adenosinergic immunosuppression during cancer immunotherapy by pharmacologically weakening the hypoxia/HIF-1 $\alpha$ axis $(7,83-86)$. The data summarized above led us to promote and pursue the elimination of the immunosuppressive hypoxia/HIF-1 $\alpha$ axis by reducing hypoxia in tumors by oxygenation. In support of this therapeutic direction were models of acute respiratory distress syndrome demonstrating the powerful oxygenation-associated exacerbation of inflammatory immune responses $(42,87)$. This led to the motivation to use oxygen to inhibit hypoxia/HIF-1 $\alpha$-mediated accumulation of extracellular adenosine in tumors, thereby preventing immunosuppression and promoting destruction of cancerous tissues $(8,80,88)$.

Preclinical biochemical studies established that intratumoral hypoxia and HIF- $1 \alpha$ govern expression of CD39 and CD73 and accumulation of tumor-protecting extracellular adenosine in the $\operatorname{TME}(8,9)$. Indeed, respiratory hyperoxia was shown to decrease intratumoral hypoxia, HIF-1 $\alpha$, and HIF- $1 \alpha$ downstream target proteins (9). This reversal of hypoxia was accompanied by the downregulation of CD39 and CD73 and reduced levels of ade- 
nosine in the TME. An immunologically relevant discovery in these studies was that systemic oxygenation upregulated antigenpresenting MHC class I molecules on tumor cells and led to better recognition and increased susceptibility to killing by tumor-reactive cytotoxic T cells (9). Follow-up studies using three-dimensional cultures that better mimic the hypoxia/adenosinergic TME established that hypoxia negatively regulates MHC expression in a HIF-dependent manner (89). These mechanistic findings confirmed that MHC expression was decreased in human renal cell carcinomas that had constitutive expression of HIF due to the genetic loss of von Hippel-Lindau (VHL) function (89). Taken together, these data revealed a mechanism by which the hypoxia/ HIF-1 $\alpha /$ A2AR axis facilitates tumor immune escape.

In parallel studies, we focused on the immunological effects of supplemental oxygen by demonstrating that the reversal of hypoxia converts an immunosuppressive TME toward immunopermission (8). Respiratory hyperoxia enhanced intratumoral infiltration of both endogenously developed and adoptively transferred tumor-reactive $\mathrm{T}$ cells in mice. This coincided with the supplemental oxygen-induced increase in the expression of a number of proinflammatory cytokines and chemokines (e.g., IL-2, IL-12, IFN- $\gamma$ ) and the downregulation of immunosuppressive molecules and Tregs (8). Of therapeutic value, respiratory hyperoxia dramatically improved tumor regression and long-term survival of mice, which was shown to be entirely dependent on T and NK cells (10). Importantly, these immunoenhancing effects of respiratory hyperoxia improved the therapeutic efficacy of adoptive cell transfer protocols ( $\mathrm{T}$ and NK cells) and dual blockade of the immune checkpoints (CTLA-4 and PD-1) (8). Taken together, these data demonstrate that HIF-1 $\alpha$ inhibits adaptive immune cells and acts as an upstream mediator of the hypoxia/adenosinergic pathway of immunosuppression. These studies were the first to provide proof of principle for using oxygen or oxygenation agents to prevent hypoxia/HIF-1 $\alpha$ /adenosine/A2AR-mediated immunosuppression and improve existing cancer immunotherapies (7-9, 80).

In pioneering studies to translate this approach into the clinic, Curran and coauthors $(18,90)$ confirmed and extended our observations of the effects of hypoxia elimination on better outcomes of existing immunotherapies $(7-9,57,58,80,86)$. The original observations that $\mathrm{T}$ cells avoid the hypoxic TME (8) were strengthened by their findings showing that hypoxic zones in prostate tumors were void of $\mathrm{T}$ cells, even after treatment with CTLA-4/PD-1 blockade (17). Authors demonstrated that the hypoxia-activated prodrug TH-302 reduces or eliminates hypoxia in tumors. This effect synergized with checkpoint blockade to cure the majority of spontaneously arising adenocarcinoma of the mouse prostate by enabling infiltration of T cells into hypoxic TMEs. Thus, there now exists sufficient evidence to expect that the reversal of hypoxia might restore $\mathrm{T}$ cell infiltration and improve sensitivity to $\mathrm{T}$ cell checkpoint blockade in cold tumors $(8,17)$.

Under the clinical leadership of David Hong, Curran's team launched a clinical trial of the combination of TH-302 and ipilimumab (anti-CTLA-4) in 2017 (ClinicalTrials.gov NCT03098160), which has revealed the potential of this combination to regress both established metastatic prostate cancer and anti-PD-1-refractory head and neck cancer in patients, as well as to provide longterm control of advanced pancreatic cancer (90). This phase I trial was halted after the dose escalation phase due to demonstration of efficacy so that a large, potentially registrational phase II study (also including an anti-PD-1 arm) could be launched in 2020. Importantly, no additive toxicity was observed between CTLA-4 blockade and TH-302 at doses up to $640 \mathrm{mg} / \mathrm{m}^{2}$ (90).

\section{Repurposing oxygenation agents for cancer immunotherapy}

While respiratory hyperoxia was shown to be an effective method to eliminate the hypoxia/HIF-1 $\alpha$ /adenosine/A2AR axis, there are obvious limitations for oxygen transport and delivery farther away from blood vessels in the pathologically chaotic TME. For this reason, we have suggested combining oxygen and oxygenation agents with cancer immunotherapy protocols (29). We propose that synthetic oxygenation agents and natural blood substitutes are powerful tools that can be repurposed to maximize the tumor-rejecting capacity of current cancer immunotherapies.

A major class of these drugs are perfluorocarbons (PFCs) highly stable synthetic molecules consisting of carbon and fluorine capable of dissolving high amounts of oxygen. First-generation PFCs (e.g., Fluosol and Perftoran) were safe at high doses in over 2000 patients in clinical trials (91). However, the design and formulation were substantially improved in second-generation PFCs, with particle sizes less than $200 \mathrm{~nm}$ to allow PFCs to enter very thin blood vessels and unload oxygen in areas of low oxygen tension, relevant to the TME (91).

We suggest that PFCs and other oxygenation agents might be an effective approach to improve cancer immunotherapy by eliminating the hypoxia/HIF-1 $\alpha /$ A2AR axis in the TME. An important consideration regarding PFCs is that the oxygen-carrying capacity is increased by higher fractions of inspired oxygen $\left(\mathrm{F}_{\mathrm{i}} \mathrm{O}_{2}\right)$. Therefore, combination of respiratory hyperoxia and oxygenation agents, like PFCs, may synergize to improve tumor regression in solid tumors - especially tumors located in distal sites.

\section{Acknowledgments}

The authors acknowledge the 20 years of generous financial support by the intramural program of the National Institute of Allergy and Infectious Diseases, NIH, and funding from the New England Inflammation and Tissue Protection Institute, Northeastern University, as well as discussions with Bruce Spiess, Department of Anesthesiology, University of Florida.

Address correspondence to: Michail V. Sitkovsky or Stephen M. Hatfield, New England Inflammation and Tissue Protection Institute, Department of Pharmaceutical Sciences and Biology, Northeastern University, 360 Huntington Avenue, Boston, Massachusetts 02115, USA. Phone: 617.373.4157; Email: m.sitkovsky@neu.edu (MVS). Phone: 617.373.5737; Email: s.hatfield@neu.edu (SMH).

\footnotetext{
1. Hellstrom KE, Hellstrom I. From the Hellstrom paradox toward cancer cure. Prog Mol Biol Transl Sci. 2019;164:1-24
}

2. Hellstrom I, Hellstrom KE, Pierce GE, Yang JP. Cellular and humoral immunity to different types of human neoplasms. Nature.
1968;220(5174):1352-1354.

3. Ohta A, Sitkovsky M. Role of G-protein-coupled

adenosine receptors in downregulation of inflam- 
mation and protection from tissue damage.

Nature. 2001;414(6866):916-920.

4. Ohta A, et al. A2A adenosine receptor protects tumors from antitumor T cells. Proc Natl Acad Sci U S A. 2006;103(35):13132-13137.

5. Sitkovsky MV. Lessons from the A2A adenosine receptor antagonist-enabled tumor regression and survival in patients with treatment-refractory renal cell cancer. Cancer Discov. 2020;10(1):16-19.

6. Fong L, et al. Adenosine $2 \mathrm{~A}$ receptor blockade as an immunotherapy for treatment-refractory renal cell cancer. Cancer Discov. 2020;10(1):40-53.

7. Sitkovsky MV, Hatfield S, Abbott R, Belikoff B, Lukashev D, Ohta A. Hostile, hypoxia-A2adenosinergic tumor biology as the next barrier to overcome for tumor immunologists. Cancer Immunol Res. 2014;2(7):598-605.

8. Hatfield SM, et al. Immunological mechanisms of the antitumor effects of supplemental oxygenation. Sci Transl Med. 2015;7(277):277ra30.

9. Hatfield SM, et al. Systemic oxygenation weakens the hypoxia and hypoxia inducible factor $1 \alpha$-dependent and extracellular adenosine-mediated tumor protection. J Mol Med (Berl). 2014;92(12):1283-1292.

10. Sitkovsky MV. T regulatory cells: hypoxiaadenosinergic suppression and re-direction of the immune response. Trends Immunol. 2009;30(3):102-108.

11. Lan J, Lu H, Samanta D, Salman S, Lu Y, Semenza GL. Hypoxia-inducible factor 1-dependent expression of adenosine receptor $2 \mathrm{~B}$ promotes breast cancer stem cell enrichment. Proc Natl Acad Sci US A. 2018;115(41):E9640-E9648.

12. Wei SC, Duffy CR, Allison JP. Fundamental mechanisms of immune checkpoint blockade therapy. Cancer Discov. 2018;8(9):1069-1086.

13. Chamoto K, Hatae R, Honjo T. Current issues and perspectives in $\mathrm{PD}-1$ blockade cancer immunotherapy. Int J Clin Oncol. 2020;25(5):790-800.

14. Daassi D, Mahoney KM, Freeman GJ. The importance of exosomal PDL1 in tumour immune evasion. Nat Rev Immunol. 2020;20(4):209-215.

15. Rosenberg SA, Restifo NP, Yang JC, Morgan RA, Dudley ME. Adoptive cell transfer: a clinical path to effective cancer immunotherapy. Nat Rev Cancer. 2008;8(4):299-308.

16. Dougan M, Dranoff G, Dougan SK. Cancer Immunotherapy: Beyond Checkpoint Blockade. Annu Rev Cancer Biol. 2019;3(1):55-75.

17. Jayaprakash $P$, et al. Targeted hypoxia reduction restores $\mathrm{T}$ cell infiltration and sensitizes prostate cancer to immunotherapy. JClin Invest. 2018;128(11):5137-5149.

18. Zarek PE, et al. A2A receptor signaling promotes peripheral tolerance by inducing $\mathrm{T}$-cell anergy and the generation of adaptive regulatory $\mathrm{T}$ cells. Blood. 2008;111(1):251-259.

19. Beavis PA, et al. Adenosine receptor $2 \mathrm{~A}$ blockade increases the efficacy of anti-PD-1 through enhanced antitumor T-cell responses. Cancer Immunol Res. 2015;3(5):506-517.

20. Young A, et al. Co-inhibition of CD73 and A2AR adenosine signaling improves anti-tumor immune responses. Cancer Cell. 2016;30(3):391-403.

21. Loi S, et al. CD73 promotes anthracycline resistance and poor prognosis in triple negative breast cancer. Proc Natl Acad Sci USA.
2013;110(27):11091-11096.

22. Palucka AK, Coussens LM. The basis of oncoimmunology. Cell. 2016;164(6):1233-1247.

23. Newick K, O’Brien S, Moon E, Albelda SM. CAR T cell therapy for solid tumors. Annu Rev Med. 2017;68:139-152.

24. van der Leun AM, Thommen DS, Schumacher TN. CD8 ${ }^{+} \mathrm{T}$ cell states in human cancer: insights from single-cell analysis. Nat Rev Cancer. 2020;20(4):218-232.

25. Semenza GL. Pharmacologic targeting of hypoxiainducible factors. Annu Rev Pharmacol Toxicol. 2019;59:379-403.

26. Ivan M, Kaelin WG. The EGLN-HIF O $\mathrm{O}_{2}$-sensing system: multiple inputs and feedbacks. Mol Cell. 2017;66(6):772-779.

27. Bullen JW, et al. Protein kinase A-dependent phosphorylation stimulates the transcriptional activity of hypoxia-inducible factor 1. Sci Signal. 2016;9(430):ra56.

28. Haskó G, Szabó C, Németh ZH, Kvetan V, Pastores SM, Vizi ES. Adenosine receptor agonists differentially regulate IL-10, TNF-alpha, and nitric oxide production in RAW 264.7 macrophages and in endotoxemic mice. JImmunol. 1996;157(10):4634-4640.

29. Ohta A, Sitkovsky M. Methods for using extracellular adenosine inhibitors and adenosine receptor inhibitors to enhance immune response and inflammation. US Patent US10314908B2. https://patents.google.com/ patent/US8716301B2/en. Updated June 11, 2019. Accessed August 20, 2020.

30. Takayama H, Sitkovsky MV. Antigen receptorregulated exocytosis in cytotoxic T lymphocytes. JExp Med.1987;166(3):725-743.

31. Trenn G, Takayama H, Sitkovsky MV. Exocytosis of cytolytic granules may not be required for target cell lysis by cytotoxic T-lymphocytes. Nature. 1987;330(6143):72-74.

32. Sitkovsky MV, Trenn G, Takayama H. Cyclic AMP-dependent protein kinase as a part of the possible down-regulating pathway in the antigen receptor-regulated cytotoxic T lymphocyte conjugate formation and granule exocytosis. Ann N Y Acad Sci. 1988;532:350-358.

33. Sitkovsky MV. Mechanistic, functional and immunopharmacological implications of biochemical studies of antigen receptor-triggered cytolytic T-lymphocyte activation. Immunol Rev. 1988;103:127-160.

34. Bjørgo E, Moltu K, Taskén K. Phosphodiesterases as targets for modulating T-cell responses. Handb Exp Pharmacol. 2011;(204):345-363.

35. Eltzschig HK, Sitkovsky MV, Robson SC. Purinergic signaling during inflammation. $N$ Engl J Med. 2013;368(13):1260.

36. Fredholm BB, Johansson S, Wang YQ. Adenosine and the regulation of metabolism and body temperature. Adv Pharmacol. 2011;61:77-94.

37. Chen JF, Eltzschig HK, Fredholm BB. Adenosine receptors as drug targets-what are the challenges? Nat Rev Drug Discov. 2013;12(4):265-286.

38. Huang S, Apasov S, Koshiba M, Sitkovsky M. Role of A2a extracellular adenosine receptor-mediated signaling in adenosine-mediated inhibition of T-cell activation and expansion. Blood. 1997;90(4):1600-1610.
39. Apasov SG, Koshiba M, Chused TM, Sitkovsky MV. Effects of extracellular ATP and adenosine on different thymocyte subsets: possible role of ATP-gated channels and $G$ protein-coupled purinergic receptor. JImmunol. 1997;158(11):5095-5105

40. Koshiba M, Rosin DL, Hayashi N, Linden J, Sitkovsky MV. Patterns of A2A extracellular adenosine receptor expression in different functional subsets of human peripheral T cells. Flow cytometry studies with anti-A2A receptor monoclonal antibodies. Mol Pharmacol. 1999;55(3):614-624.

41. Armstrong JM, et al. Gene dose effect reveals no Gs-coupled A2A adenosine receptor reserve in murine T-lymphocytes: studies of cells from A2A-receptor-gene-deficient mice. Biochem J. 2001;354(pt 1):123-130.

42. Thiel M, et al. Oxygenation inhibits the physiological tissue-protecting mechanism and thereby exacerbates acute inflammatory lung injury. PLoS Biol. 2005;3(6):e174.

43. Kaczmarek E, et al. Identification and characterization of CD39/vascular ATP diphosphohydrolase. J Biol Chem. 1996;271(51):33116-33122.

44. Sun X, et al. CD39/ENTPD1 expression by CD4+Foxp3+ regulatory $\mathrm{T}$ cells promotes hepatic metastatic tumor growth in mice. Gastroenterology. 2010;139(3):1030-1040.

45. Jin D, et al. CD73 on tumor cells impairs antitumor T-cell responses: a novel mechanism of tumor-induced immune suppression. Cancer Res. 2010;70(6):2245-2255.

46. Stagg J, et al. Anti-CD73 antibody therapy inhibits breast tumor growth and metastasis. Proc Natl Acad Sci U S A. 2010;107(4):1547-1552.

47. Eckle T, et al. Identification of ectonucleotidases CD39 and CD73 in innate protection during acute lung injury. J Immunol. 2007;178(12):8127-8137.

48. Grenz A, et al. Contribution of E-NTPDase1 (CD39) to renal protection from ischemia-reperfusion injury. FASEB J. 2007;21(11):2863-2873.

49. Colgan SP, Eltzschig HK, Eckle T, Thompson LF. Physiological roles for ecto-5'-nucleotidase (CD73). Purinergic Signal. 2006;2(2):351-360.

50. Antonioli L, Blandizzi C, Pacher P, Hasko G. Immunity, inflammation and cancer: a leading role for adenosine. Nat Rev Cancer. 2013;13(12):842-857.

51. Synnestvedt K, et al. Ecto-5'-nucleotidase (CD73) regulation by hypoxia-inducible factor- 1 mediates permeability changes in intestinal epithelia. J Clin Invest. 2002;110(7):993-1002.

52. Kojima H, et al. Abnormal B lymphocyte development and autoimmunity in hypoxia-inducible factor 1alpha -deficient chimeric mice. Proc Natl Acad Sci U S A. 2002;99(4):2170-2174.

53. Lukashev D, et al. Cutting edge: hypoxia-inducible factor 1alpha and its activation-inducible short isoform I.1 negatively regulate functions of CD4+ and CD8+ T lymphocytes. JImmunol. 2006;177(8):4962-4965.

54. Lukashev D, Caldwell C, Ohta A, Chen P, Sitkovsky M. Differential regulation of two alternatively spliced isoforms of hypoxia-inducible factor-1 alpha in activated T lymphocytes. JBiol Chem. 2001;276(52):48754-48763.

55. Ramanathan M, et al. Differential regulation of HIF-1alpha isoforms in murine macrophages by 
TLR4 and adenosine $\mathrm{A}(2 \mathrm{~A})$ receptor agonists JLeukoc Biol. 2009;86(3):681-689.

56. Lukashev D, Sitkovsky M. Preferential expression of the novel alternative isoform I.3 of hypoxiainducible factor 1alpha in activated human T lymphocytes. Hum Immunol. 2008;69(7):421-425.

57. Georgiev P, Belikoff BG, Hatfield S, Ohta A, Sitkovsky MV, Lukashev D. Genetic deletion of the HIF-1 $\alpha$ isoform I.1 in T cells enhances antibacterial immunity and improves survival in a murine peritonitis model. Eur JImmunol. 2013;43(3):655-666.

58. Thiel M, et al. Targeted deletion of HIF-1alpha gene in $\mathrm{T}$ cells prevents their inhibition in hypoxic inflamed tissues and improves septic mice survival. PLoS One. 2007;2(9):e853.

59. Kjaergaard J, Hatfield S, Jones G, Ohta A, Sitkovsky $\mathrm{M} . \mathrm{A}_{2 \mathrm{~A}}$ adenosine receptor gene deletion or synthetic $\mathrm{A}_{2 \mathrm{~A}}$ antagonist liberate tumor-reactive $\mathrm{CD} 8^{+} \mathrm{T}$ cells from tumor-induced immunosuppression. J Immunol. 2018;201(2):782-791.

60. Ohta A, et al. In vitro induction of $\mathrm{T}$ cells that are resistant to $\mathrm{A} 2$ adenosine receptormediated immunosuppression. Br J Pharmacol. 2009;156(2):297-306.

61. Waickman AT, Alme A, Senaldi L, Zarek PE, Horton M, Powell JD. Enhancement of tumor immunotherapy by deletion of the A2A adenosine receptor. Cancer Immunol Immunother. 2012;61(6):917-926.

62. Beavis PA, Milenkovski N, Stagg J, Smyth MJ, Darcy PK. $A_{2 A}$ blockade enhances anti-metastatic immune responses. Oncoimmunology. 2013;2(12):e26705.

63. Beavis PA, et al. Blockade of A2A receptors potently suppresses the metastasis of CD73+ tumors. Proc Natl Acad Sci U S A. 2013;110(36):14711-14716.

64 . Beavis PA, et al. Targeting the adenosine $2 \mathrm{~A}$ receptor enhances chimeric antigen receptor $\mathrm{T}$ cell efficacy. J Clin Invest. 2017;127(3):929-941.

65. Sheridan C. Adorx dives into the adenosine antagonism pool with $\$ 10 \mathrm{M}$ series A. BioWorld. 2018;29(121):1-6.

66. Willingham SB, et al. A2AR antagonism with CPI-444 induces antitumor responses and augments efficacy to anti-PD-(L) 1 and anti-CTLA-4 in preclinical models. Cancer Immunol Res. 2018;6(10):1136-1149.

67. Caldwell CC, et al. Differential effects of physiologically relevant hypoxic conditions on $\mathrm{T}$ lymphocyte development and effector functions. Jimmunol. 2001;167(11):6140-6149.

68. Kojima H, et al. Differentiation stage-specific requirement in hypoxia-inducible factor-1alpharegulated glycolytic pathway during murine $B$ cell development in bone marrow. Jimmunol. 2010;184(1):154-163.

69. Abbott RK, et al. Germinal center hypoxia potentiates immunoglobulin class switch recombination. J Immunol. 2016;197(10):4014-4020.

70. Cho SH, et al. Germinal centre hypoxia and regulation of antibody qualities by a hypoxia response system. Nature. 2016;537(7619):234-238.

71. Cho SH, et al. Hypoxia-inducible factors in $\mathrm{CD}^{+}{ }^{+}$ cells promote metabolism, switch cytokine secretion, and T cell help in humoral immunity. Proc Natl Acad Sci U S A . 2019;116(18):8975-8984.

72. Ohta A, Diwanji R, Kini R, Subramanian M, Ohta A, Sitkovsky M. In vivo T cell activation in lymphoid tissues is inhibited in the oxygen-poor microenvironment. Front Immunol. 2011;2:27.

73. Choukèr A, et al. Critical role of hypoxia and A2A adenosine receptors in liver tissue-protecting physiological anti-inflammatory pathway. $\mathrm{Mol}$ Med. 2008;14(3-4):116-123.

74. Choukèr A, et al. In vivo hypoxic preconditioning protects from warm liver ischemia-reperfusion injury through the adenosine $\mathrm{A} 2 \mathrm{~B}$ receptor. Transplantation. 2012;94(9):894-902.

75. Srinivasan S, et al. Sphingosine-1-phosphate reduces CD4+ T-cell activation in type 1 diabetes through regulation of hypoxia-inducible factor short isoform I.1 and CD69. Diabetes. 2008;57(2):484-493.

76. Ohta A, et al. Hypoxia-induced and A2A adenosine receptor-independent $\mathrm{T}$-cell suppression is short lived and easily reversible. Int Immunol. 2014;26(2):83-91.

77. Palazon A, et al. An HIF-1 $\alpha /$ VEGF-A axis in cytotoxic $\mathrm{T}$ cells regulates tumor progression. Cancer Cell. 2017;32(5):669-683.

78. Wong CC, et al. Inhibitors of hypoxia-inducible factor 1 block breast cancer metastatic niche formation and lung metastasis. J Mol Med (Berl). 2012;90(7):803-815.

79. Dewhirst MW, Cao Y, Moeller B. Cycling hypoxia and free radicals regulate angiogenesis and radiotherapy response. Nat Rev Cancer. 2008;8(6):425-437.

80. Hatfield SM, Sitkovsky M. Oxygenation to improve cancer vaccines, adoptive cell transfer and blockade of immunological negative regulators. Oncoimmunology. 2015;4(12):e1052934.

81. Schito L, Semenza GL. Hypoxia-inducible factors: master regulators of cancer progression. Trends Cancer. 2016;2(12):758-770.

82. Semenza GL. Hypoxia-inducible factors in physiology and medicine. Nobel Lecture. https://www. nobelprize.org/uploads/2019/10/semenzalecture-slides.pdf. Accessed August 20, 2020

83. Sitkovsky MV, et al. Physiological control of immune response and inflammatory tissue damage by hypoxia-inducible factors and adenosine A2A receptors. Annu Rev Immunol. 2004;22:657-682.

84. Sitkovsky M, Lukashev D. Regulation of immune cells by local-tissue oxygen tension: HIF1 alpha and adenosine receptors. Nat Rev Immunol. 2005;5(9):712-721.

85. Sitkovsky MV, Kjaergaard J, Lukashev D, Ohta A. Hypoxia-adenosinergic immunosuppression: tumor protection by $\mathrm{T}$ regulatory cells and cancerous tissue hypoxia. Clin Cancer Res. 2008;14(19):5947-5952.

86. Sitkovsky M, Ohta A. Targeting the hypoxiaadenosinergic signaling pathway to improve the adoptive immunotherapy of cancer. J Mol Med (Berl). 2013;91(2):147-155

87. Zangl Q, et al. Postoperative hyperoxia (60\%) worsens hepatic injury in mice. Anesthesiology. 2014;121(6):1217-1225

88. Lukashev D, Ohta A, Sitkovsky M. Hypoxia-dependent anti-inflammatory pathways in protection of cancerous tissues. Cancer Metastasis Rev. 2007;26(2):273-279.

89. Sethumadhavan S, et al. Hypoxia and hypoxiainducible factor (HIF) downregulate antigenpresenting $\mathrm{MHC}$ class I molecules limiting tumor cell recognition by T cells. PLoS One. 2017;12(11):e0187314.

90. Hegde A, et al. A phase 1 dose escalation study to evaluate the safety and tolerability of evofosfamide in combination with ipilimumab in advanced solid malignancies. Poster presented at: Society for Immunotherapy of Cancer 34th Annual Meeting; National Harbor, Maryland, USA; November 6-10, 2019. https://sitc.sitcancer.org/2019/abstracts/titles/index.php?filter=Clinical. Accessed August 20, 2020.

91. Winslow RM. Blood substitutes. Curr Opin Hematol. 2002;9(2):146-151. 